\title{
Surveying Phylogenetic Footprints in Large Gene Clusters: Applications to Hox Cluster Duplications
}

\author{
Sonja Prohaska ${ }^{\mathrm{a}, \mathrm{b}}$, Claudia Fried ${ }^{\mathrm{a}, \mathrm{b}}$, Christoph Flamm ${ }^{\mathrm{b}}$, \\ Günter P. Wagner ${ }^{c}$, Peter F. Stadler ${ }^{\mathrm{a}, \mathrm{b}, \mathrm{d}}$ \\ ${ }^{a}$ Lehrstuhl für Bioinformatik, Institut für Informatik, Universität Leipzig, \\ Kreuzstraße 7b, D-04103 Leipzig, Germany. \\ \{sonja, claudia, studla\}@bioinf.uni-leipzig.de \\ ${ }^{\mathrm{b}}$ Institut für Theoretische Chemie und Molekulare Strukturbiologie, \\ Universität Wien, Währingerstraße 17, A-1090 Wien, Austria \\ \{sopr, claudia, xtof, studla\}@tbi.univie.ac.at \\ ${ }^{\mathrm{c}}$ Department of Ecology and Evolutionary Biology \\ Yale University, New Haven, CT, USA \\ gunter.wagner@yale.edu \\ ${ }^{\mathrm{d}}$ The Santa Fe Institute, 1399 Hyde Park Road, Santa Fe, NM 87501, USA
}

\begin{abstract}
Evolutionarily conserved non-coding genomic sequences represent a potentially rich source for the discovery of gene regulatory regions. Since these elements are subject to stabilizing selection they evolve much slower than adjacent non-functional DNA. These so-called phylogenetic footprints can be detected by comparison of the sequences surrounding orthologous genes in different species. In this paper we present a new method and an efficient software tool for the identification of corresponding footprints in long sequences from multiple species. This allows the evolutionary study of the origin and loss of phylogenetic footprints if sufficient number and appropriately placed species are included. We apply this method to the published sequences of HoxA clusters of shark, human, and the duplicated zebrafish and Takifugu clusters as well as the published HoxB cluster sequences. We find that there is a massive loss of sequence conservation in the intergenic region of the HoxA clusters, consistent with the finding in [Chiu et al., PNAS 99, 5492-5497 (2002)]. We further propose a simple model to estimate the loss of sequence conservation that can be attributed to gene loss and other structural reasons. We find that the loss of conservation after cluster duplication is more extensive than expected by this model. This suggests that binding site turnover and/or adaptive modification may also contribute to the loss of sequence conservation. We conclude that this method is suitable for the large scale study of the evolution of (putative) cis-regulatory elements.
\end{abstract}

Key words: Phylogenetic footprints, Hox gene clusters, gene duplication 


\section{Introduction}

Non-coding DNA in eukaryotes contains a large number functionally important signals for the regulation of gene expression. These cis-acting regulatory elements can be interpreted as the "hardwiring of development" at the genomic level [4]. For a recent review see [20].

Functional and non-functional parts of genomes evolve with different speeds reflecting the fact that mutations are selected against in the functional parts [17]. The technique of Phylogenetic Footprinting exploits these differential evolution rates for identifying regulatory elements [29].

There are two classes of approaches to identify regulatory regions. Most commonly, one searches for common motifs in the non-coding sequences associated with related genes in the same organism, see e.g. [15,27,31]. Alternatively, orthologous non-coding sequences from a group of related species are used. Unusually well-conserved sequences then hint at a regulatory function. This approach was successful to identify the regulatory elements in many cases, see e.g. $[18,23,29,9,6]$ and the review [11]. In a related approach, the rVISTA tool uses pairwise alignments of orthologous regions to determine the significance of putative transcription factor binding sites found by comparison with a database of binding motifs [19]. Most searches for phylogenetic footprints in the past were based on computing global alignments. Standard motif search techniques such as AlignAce [16] and ANN-Spec [32] and segment-based alignment algorithm such as DIALIGN [24] have been shown to be more efficient [5]. Most recently footprinting was expressed as a substring parsimony problem and an exact and rather efficient dynamic programming algorithm was proposed and implemented [5]. This method takes the known phylogeny of the involved species explicitly into account and retrieves all common substrings with a better-than-threshold parsimony score from a set of input sequences.

In this contribution we pursue a different algorithmic approach that appears to be more suitable for large clusters of genes with complex regulation structure such as the Hox clusters. The reason is that at least in this case there appear to be substantial changes in the regulatory patterns that do not necessarily conform with established phylogenetic relationships: In [9], for example, it has been reported that - quite unexpectedly - the footprint pattern of the horn shark Heterodontus francisci has much more in common with the pattern in Homo sapiens than with other fish species (Morone saxatilis and Danio rerio). We therefore drop the maximum parsimony assumption for the evolution of regulatory sequences in large gene clusters and instead adopt a stepwise procedure that first extracts potentially conserved regions from pairwise sequence comparisons and passes these candidates through a series of filtering steps. Since our software tracker is intended for large-scale surveys of 
large gene clusters the entire procedure has been fully automated and includes a variety of post processing and analysis steps, in particular the assembly of regions that contain footprints in various combinations of sequences to multiple sequence alignments.

As a first application of our technique we re-evaluate and extend the survey of the HoxA clusters of Heterodontus francisci, Morone saxatilis, Danio rerio and Homo sapiens. Our main result is that the automatized procedure detects a more complete set of footprints, and that it does so in less than a minute on a modern PC, in contrast to weeks of tedious analysis with web-based bioinformatics tools.

We then extend the analysis to include the two HoxA clusters of Takifugu, based on the published genomic sequence [34], to assess whether the new method provides biologically meaningful and consistent results. The purpose of the study [9] was to assess the effect of Hox cluster duplication on the structure and function of Hox genes. The qualitative results in [9] suggested that cluster duplication leads to a massive loss of non-coding sequence conservation, which could be indicative of extensive modifications in the function of Hox genes. If this is the case one would expect to find a similar degree of loss of conservation in other teleost Hox clusters. In fact we do find an even greater loss of sequence conservation in Takifugu than in zebra fish (see below).

The quantitative analysis of the retention statistic for phylogenetic footprint clusters (PFCs) has to be put into context of the other changes that happen after Hox cluster duplication. Most notably there is a tendency for gene loss after duplication [1] which can have a direct, "structural" rather than functional, influence on the retention of PFCs. To assess whether the loss of sequence conservation can be explained in its entirety by gene loss we propose a simple probabilistic model for the rate of PFC loss due to gene loss and stochastic resolution of genetic redundancy (structural causes). With this model we show that the number of PFC retained is less than what is expected from structural causes in all examined cases. This supports the idea that Hox cluster duplication can facilitate the evolution of development $[22,9]$.

\section{Materials}

A whole-genome shotgun assembly of the genome of Takifugu rubripes was published recently [2]. Blast searches of the known Hox-A proteins from other species against version 3.0 of the Fugu database [34] leave little doubt that there are two Hox-A clusters. The Hox-Aa cluster is located in scaffold 47. It differs from its zebrafish-homologue in two features: (a) Takifugu has a Hox-10 and (b) the Hox-2 gene is retained in Takifugu. The best homologues 


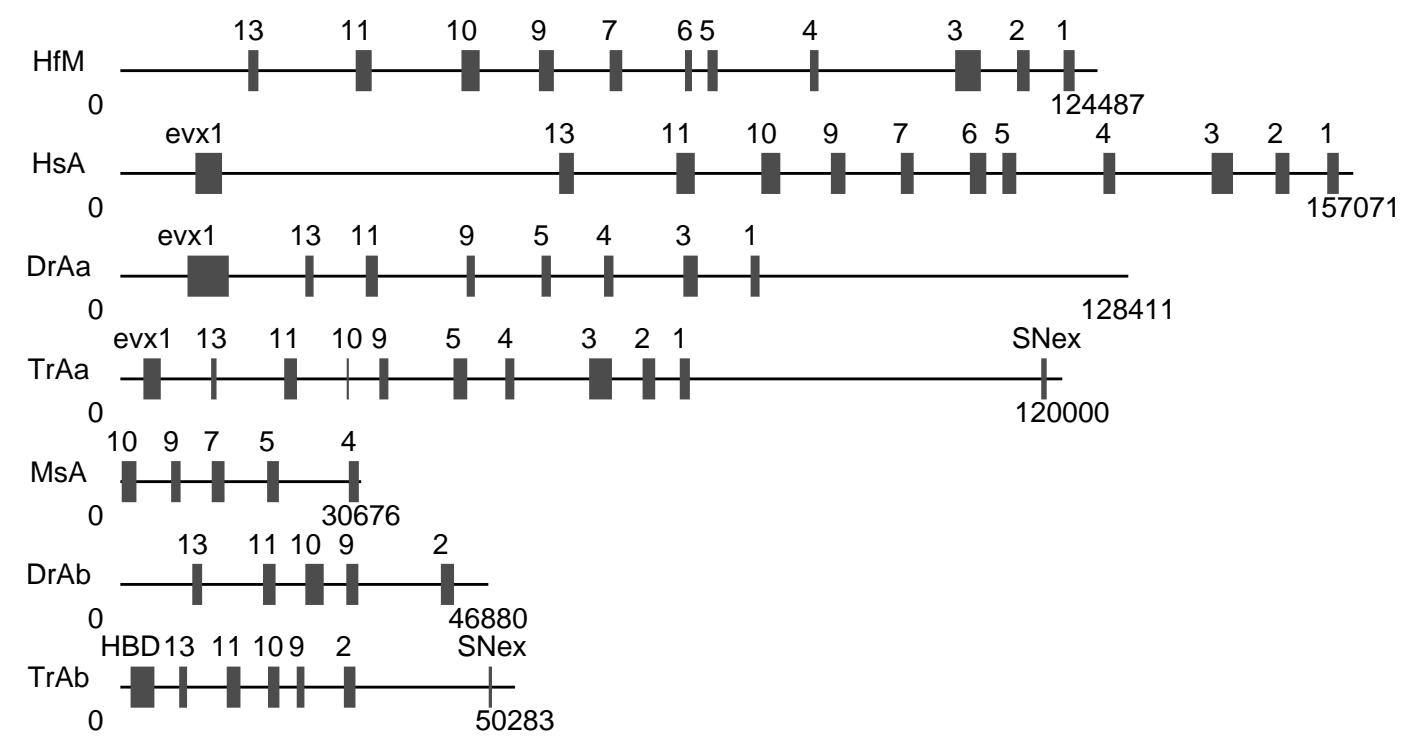

Fig. 1. Hox-A clusters used in this paper. Accession numbers and data sources:

HsA Homo sapiens AC004080 (reverse complement), AC010990 (r.c., overlaps 200nt with AC004080), and AC004079 (pos. 75001-end, r.c., overlaps 200nt with AC010990), as in [9];

HfM Heterodontus francisici AF479755 (as in [9]);

DrAa Danio rerio HoxAa: AC107365 (reverse complement);

DrAb Danio rerio HoxAb: AC107364 the first 50000nt are omitted in this drawing; TrAa Takifugu rubripes HoxAa: Fugu v.3.0 scaffold 47 positions 103001-223000 (reverse complement), contains FRU92573

TrAb Takifugu rubripes HoxAb: Fugu v.2.0 scaffold 1874

MsAMorone saxatilis AF089743, almost certainly an Aa cluster.

of the Hox-Ab genes of Danio rerio are found in scaffold 330 and scaffold 5310. In the previous release 2.0 the entire Hox-Ab cluster is contained in the single scaffold 1874 . The assembly in version 2.0 is furthermore consistent with the "d-cluster" of [3]. The best blast hits for the proteins of the fugu v.3.0 gene model are almost exclusively Hox-A genes from human, horn shark, and teleost species. The gene inventory of scaffold 1874 is identical with the HoxAb cluster of the zebrafish Danio rerio, see also [26]. Sequences for Hox-B, Hox-C, and Hox-D clusters were obtained from Genbank, the Fugu database [34], and the web pages of the Zebrafish Sequencing Project [35], for further details see the caption of Figure 2. Prince [26] notes that Takifugu has most likely two A, one B, and one $\mathrm{C}$ cluster. The sequences obtained from the Fugu database [34] contain unambiguous evidence for the existence of two B-clusters in this species, see Fig. 2. Comparisons of known Hox genes with the putative hox genes of the cluster sequences are provided as supplemental material ${ }^{1}$.

$\overline{1 \text { See www bioinf .uni-leipzig.de/Publications/SUPPLEMENTS/. }}$ 


\section{Method}

\subsection{Initial set of pairwise alignments}

The program tracker is based upon blastz [28] searches of all sequences against each other. The resulting list of raw pairwise sequence alignments is then assembled into clusters of partially overlapping regions that are analyzed in detail. By default, only the intergenic regions between two homologous genes are compared. Additional (non-homologous) genes contained in one or both sequences are disregarded. For instance the IGR between Hox-A9b and Hox-A2b together with the region between Hox-A2b and SNex of Takifugu is compared with the region between Hox-A9a and SNex of the zebrafish with the exception of the exons and introns of the zebrafish Hox-A5a, Hox-A4a, Hox-A3a, and Hox-A1a genes and the Takifugu Hox-A2b gene. Formally, the combined results of all blastz comparisons of the input sequence $x^{1}, x^{2}, \ldots, x^{N}, N \geq 3$, form a set $\mathfrak{A}=\left\{A_{k} \mid k=1, \ldots, M\right\}$ of alignments which is bases of all further analysis steps.

We perform the blastz searches with non-stringent parameters in an attempt to avoid false negative at this stage. As an undesirable side-effect of reducing the stringency of blastz we observe that some repetitive sequence elements slip into the initial set of alignments. We use the rather straightforward local entropy criterion described below to identify such sequences and to remove the corresponding parts of pairwise alignments from our initial list. In some cases the repetitive sequences actually connect two significantly conserved sequences. In this case we fragment the alignment into two or more shorter ones.

Local entropy measures are based on the nucleotide frequencies $f_{a}(k)$ measured for a sequence window $[k-W / 2, k+W / 2]$ of width $W$ around position $k$. In addition, we use analogously defined joint frequencies $f_{a b}^{\tau}(k)$ of finding the nucleotides $a$ and $b$ separated by a distance $\tau$ along the chain. The corresponding local entropies are

$$
H(k)=-\sum_{a} f_{a}(k) \log _{2} f_{a}(k) \quad H_{\tau}(k)=-\sum_{a, b} f_{a b}^{\tau}(k) \log _{2} f_{a b}^{\tau}(k)
$$

Clearly, $H(k) \leq 2$ bit and $H_{\tau}(k) \leq 4$ bit. We designate a position $k$ as having "low complexity" if both $H(k)$ and the average mutual information measure

$$
M(k)=\frac{1}{\tau_{\max }} \sum_{\tau=1}^{\tau_{\max }} H_{\tau}(k)-H(k)
$$

are smaller than user-defined threshold values $H_{\min }$ and $M_{\min }$, respectively. 


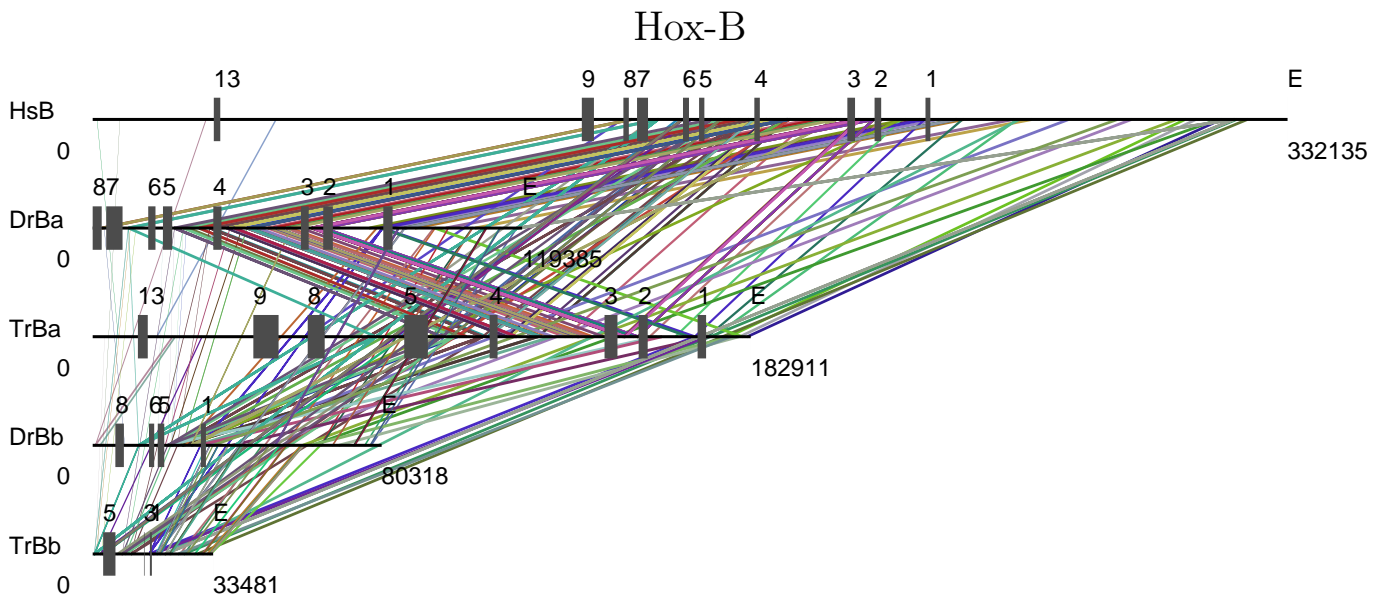

Hox-C

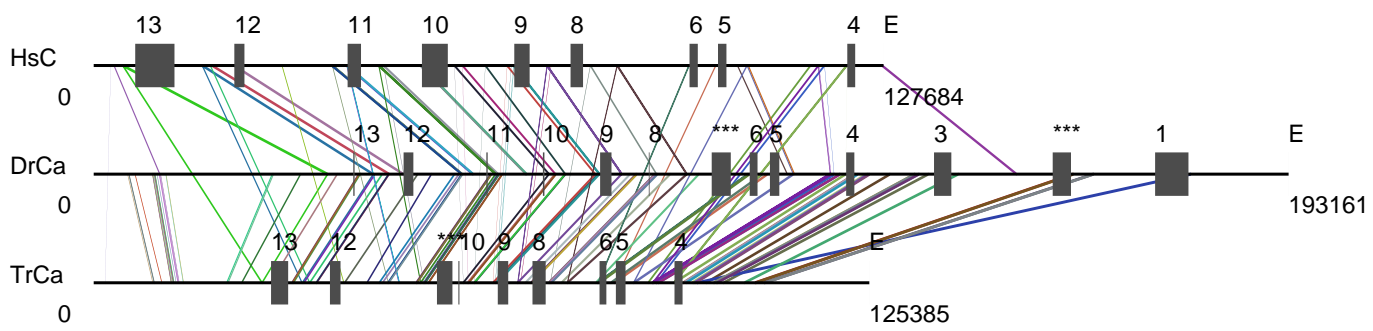

Hox-D

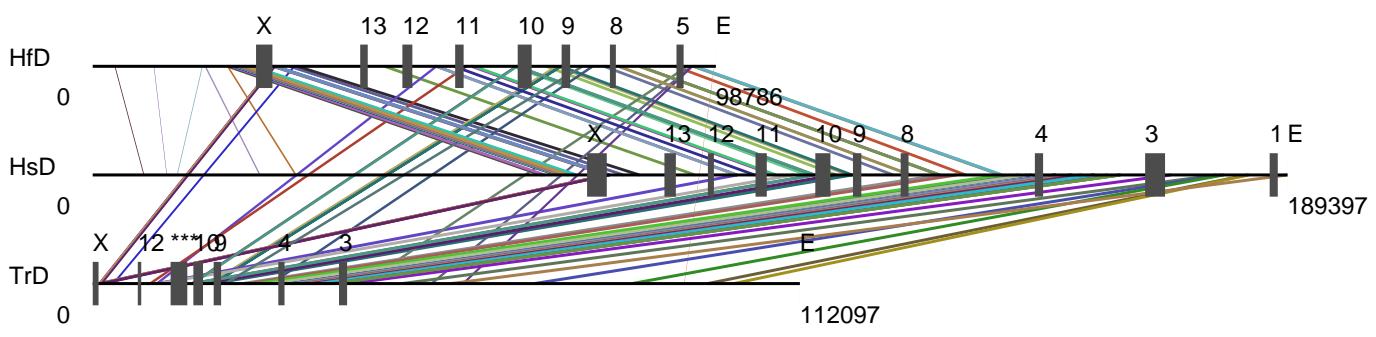

Fig. 2. Phylogenetic footprints in Hox-B, Hox-C, and Hox-D clusters. Such overviews are automatically generated by tracker. Each line corresponds to a footprint, consistent cliques (PFC) are shown with the same color. Input sequences were obtained as follows:

HsB = NT_010783 [931646-1263780] reverse complement, HsC = NT_009563 [580371-708054] r.c., HsD = NT_037537 [4075338-end]; HfD = AF224263; DrBa $=\mathrm{AL} 645782, \mathrm{DrBb}=\mathrm{AL645798}, \mathrm{DrCa}$ is a composite of zK81P22.00296(r.c.) + $3084 \times \mathrm{N}+$ zK81P22.01466(r.c.) $+2956 \times N+$ zK81P22.00552 from the Sanger site (download 12.1.03) with approximately $3000 \mathrm{Ns}$ as spacers inserted (marked by $* * *$ in the drawing); TrBa is a composite of scaffold_1439(r.c) $+2501 \times \mathrm{N}+$ scaffold_706 from version 3.0 of the Fugu DB [34], TrBb is a composite of scaffold_1245 [59047-end] $+3020 \times \mathrm{N}+$ scaffold_2182 [1-19481], $\operatorname{TrC}$ is a composite of scaffold_93[184545-end] $+2936 \times \mathrm{N}+$ scaffold_285 [134158-end] (r.c.), $\operatorname{TrD}$ is a composite of scaffold_3959 (r.c.) $+2645 \times \mathrm{N}+$ scaffold_214 [160440-end] (r.c.). All these composite sequence are consistent with a single contiguous cluster. 
Table 1

Default parameters for tracker.

\begin{tabular}{|c|c|c|c|}
\hline Processing step & \multicolumn{2}{|l|}{ Parameter } & Value \\
\hline blastz search & Minimal Score & $K$ & 1500 \\
\hline \multirow[t]{4}{*}{ Low Complexity Detection } & Window Size & $W$ & 20 \\
\hline & Separation & $\tau_{\max }$ & 6 \\
\hline & Minimal Entropy & $H_{\min }$ & 1.25 \\
\hline & Minimal Avg. Surprisal & $M_{\min }$ & 0.75 \\
\hline \multirow[t]{3}{*}{ Minimum Identity } & Window Size & $L$ & 12 \\
\hline & Quality of Best Block & $\mu_{\min }$ & $75 \%$ \\
\hline & Low Quality Cutoff & $\nu_{\max }$ & $35 \%$ \\
\hline Cluster Construction & Maximal Distance & $D_{\max }$ & 0 \\
\hline Clique Decomposition & Tolerance & $t$ & 3 \\
\hline
\end{tabular}

The second problem with the initial blastz alignments is that in many cases they consist of a few highly conserved blocks separated by relatively long (serveral dozens of nucleotides) stretches of completely diverged sequences. We therefore re-align the blastz hits using a conventional dynamic programming alignment algorithm such as clustalw [30] and post-process these alignments. We define the partial aligment $\mathcal{A}[k, l]$ as sufficiently conserved if (i) contains a sequence window $[p, p+L-1]$ of length $L$ in which the sequence identity is at least $\mu_{\min }$ and (ii) if it does not contain a window of the same length $L$ with an identity of less than $\nu_{\max }$.

\subsection{Consistent Cliques}

Each alignment $A_{k}=\left\{x^{p}[i . . j], x^{q}[k . . l]\right\}$ is represented as pair of intervals $A_{k}=\left\{A_{k}^{1}, A_{k}^{2}\right\}=\left\{x^{p}[i . . j], x^{q}[k . . l]\right\}$ where $A_{k}^{1} x^{p}[i . . j]$ denotes the subsequence of input sequence $x^{p}$ from positions $i$ to $j$. For short, we will often write $A_{k}=\left[p_{i}, p_{j}\right],\left[q_{k}, q_{l}\right]$ in the following.

We say that two alignments $A$ and $B$ overlap if there is a sequence interval $u=A_{k}^{1}$ or $u=A_{k}^{2}$ and a sequence interval $v=B_{k}^{1}$ or $v=B_{k}^{2}$ that "overlap", i.e., $u \cap v \neq \emptyset$. In the following steps it may be convenient to treat almost overlapping alignments, i.e., those that come closer than a small distance $D_{\max }$ on one sequence, as if they were overlapping. We can view the combined results from the blastz scans as a graph $\Gamma$ that has the individual blastz-alignments 


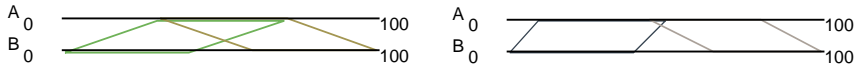

(a) (b)

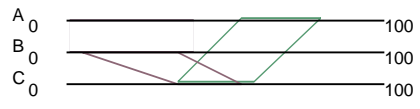

(c)

Fig. 3. (a) Two alignments that overlap in sequence $A$ match with disjoint subsequences of $B$ : clearly these to alignments are inconsistent in the sense that cannot even be approximately be part of a common alignment. (b) This situation on the r.h.s. is more subtle because the small overlap of only a few nucleotides might be the artifact here. In this case we might want to treat them as a single alignment with a long insertion in sequence $B$. (c) In this case the alignments between sequence $A-B$ and $A-C$ are inconsistent because different subsequences of $A$ are mapped to the same subsequence of $C$ by means of the $B-C$ alignment. Note that iff we were to disregard the $B-C$ then the $A-B$ and the $A-C$ alignments belong to different connected components.

as its vertices. The edges of $\Gamma$ are then the (almost) overlapping alignments.

Overlapping alignments may either indicate that (parts of) footprints are conserved between more than two sequences or they arise e.g. by the duplication of a footprint pattern in one or both of the input sequences. The second stage of a tracker run therefore consists of a careful analysis of the overlap graph and its constituent sequence alignments.

The first step is the decomposition of $\Gamma$ into its connected components $\Gamma_{i}$, $i=1, \ldots, n_{C}$, which we will refer to as "clusters". The complicated part of the analysis is of course the further investigation of the individual clusters since they may contain mutually incompatible alignments.

From the graph-theoretical point of view it seems most natural to first consider the question whether alignments within a cluster are indeed compatible with each other, or whether they are incompatible in some way. Then one may define a graph $\Psi_{i}$ that has the blastz-alignments of the cluster $\Gamma_{i}$ as its vertices and has and edge between $A$ and $B$ if and only if $A$ and $B$ are incompatible. What we really want to know are the cliques of the complement graph $\overline{\Psi_{i}}$ (which has an edge between $A$ and $B$ if and only if there is no edge in $\Psi_{i}$ ). These are efficiently computed by means of the Bron-Kerbosch algorithm [7]. It remains to specify when pairwise sequence alignments are incompatible for our purposes.

The simplest case of incompatibility involves only a pair of alignments $A=$ $\left\{x\left[i . i^{\prime}\right], y\left[j, j^{\prime}\right]\right\}$ and $B=\left\{x\left[k . . k^{\prime}\right], y\left[l, l^{\prime}\right]\right\}$ between the same two input sequences $x$ and $y$ that overlap in one sequence but not in the other one, as in the example shown in Fig. 3a,b. More complicated inconsistencies appear to be very rare in practical applications with few sequences. Below we describe 


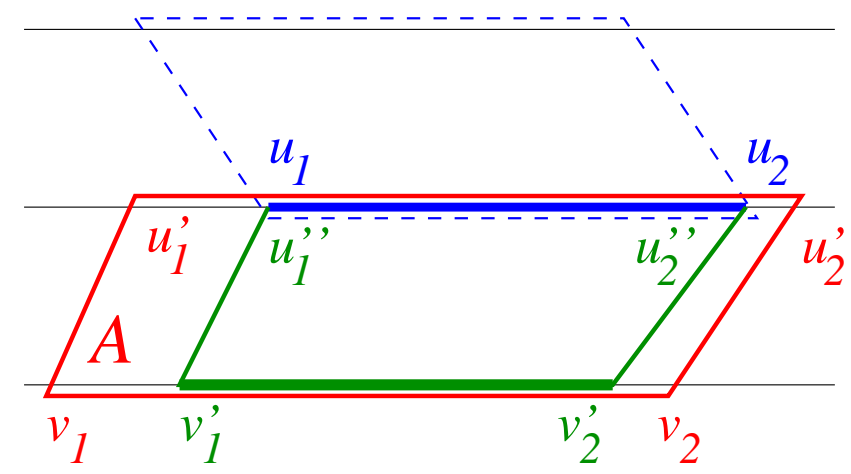

Fig. 4. Notation for the inconsistency-finding algorithm. $\left[v_{1}^{\prime}, v_{2}^{\prime}\right]$ is trace of $\left[u_{1}, u_{2}\right]$ under the alignment $A$. See text for details.

a general procedure for determining inconsistent alignments within a cluster which will be indispensible for larger sets of input data.

The basic idea is to consider a sequence of distinct alignments $A_{k}=\left\{A_{k}^{1}, A_{k}^{2}\right\}$ such that $A_{j}^{2} \cap A_{j+1}^{1} \neq \emptyset$. Any such sequence corresponds to a path in the overlap graph $\Gamma_{i}$. Then we consider the image of the initial sequence interval $A_{1}^{1}$ at each step of the sequence. Whenever $A_{k}^{2}$ and $A_{1}^{1}$ are parts of the same input sequence an inconsistency occurs if $A_{k}^{2} \nsubseteq A_{1}^{1}$, i.e., if the image of $A_{1}^{1}$ after a sequence of alignments is another interval on the same input sequence, see Fig. 3c. The sequences of alignments correspond to paths in the overlap graph $\Gamma_{i}$.

In order to find alignments in the cluster that are inconsistent with an alignment $A_{0}=\left[p_{1}, p_{2}\right],\left[q_{1}, q_{2}\right]$ build directed tree recursively starting with the directed edge $\left[p_{1}, p_{2}\right] \rightarrow\left[q_{1}, q_{2}\right]$. To each endpoint $u$ of the growing tree (except $\left[p_{1}, p_{2}\right]$, of course), which is associated with an interval $\left[u_{1}, u_{2}\right]$, we attach edges for each alignment that overlaps with $\left[u_{1}, u_{2}\right]$ and has not be used already along the path from from $\left[p_{1}, p_{2}\right]$ to $\left[u_{1}, u_{2}\right]$. The vertex at the endpoint of the new edge is associated with the interval $\left[v_{1}^{\prime}, v_{2}^{\prime}\right]$ that is defined as the part of $\left[v_{1}, v_{2}\right]$ aligned with the overlap $\left[u_{1}^{\prime \prime}, u_{2}^{\prime \prime}\right]=\left[u_{1}, u_{2}\right] \cap\left[u_{1}^{\prime}, u_{2}^{\prime}\right]$, see Fig. 4 . We call $\left[v_{1}^{\prime}, v_{2}^{\prime}\right]$ the trace of $\left[u_{1}, u_{2}\right]$ under $A_{k}$. The traces can be interpreted as sequence pieces that should be aligned with $\left[p_{1}, p_{2}\right]$ according to the sequence of alignments. If we arrive at a trace $\left[p_{1}, p_{2}\right]$ such that there is an previously constructed trace $\left[p_{1}^{\prime}, p_{2}^{\prime}\right]$ satisfying $\left[p_{1}, p_{2}\right] \subseteq\left[p_{1}^{\prime}, p_{2}^{\prime}\right]$ that we can abandon the branch at $\left[p_{1}, p_{2}\right]$.

The preprocessed alignments do not contain large gaps in our case. We can therefore estimate the traces just from the intervals by assuming that alignments act like linear transformations on the intervals. Simply determine $\alpha_{j}$ such that $u_{j}^{\prime \prime}=u_{1}^{\prime}+\alpha_{j}\left(u_{2}^{\prime}-u_{1}^{\prime}\right)$ for $j=1,2$, i.e., $\alpha_{j}=\left(u_{j}^{\prime \prime}-u_{j}^{\prime}\right) /\left(u_{2}^{\prime}-u_{1}^{\prime}\right)$; 


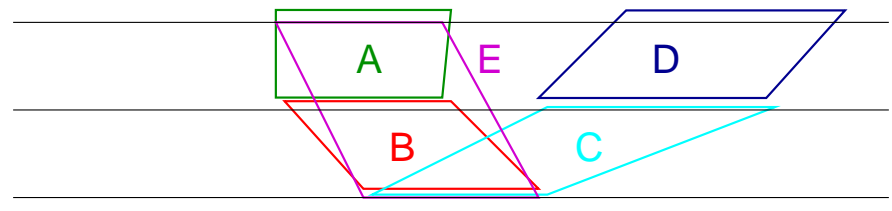

Alignments

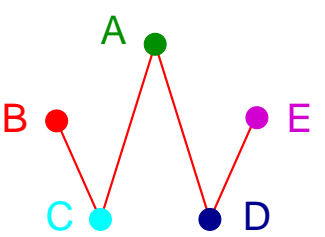

incompatibility

graph
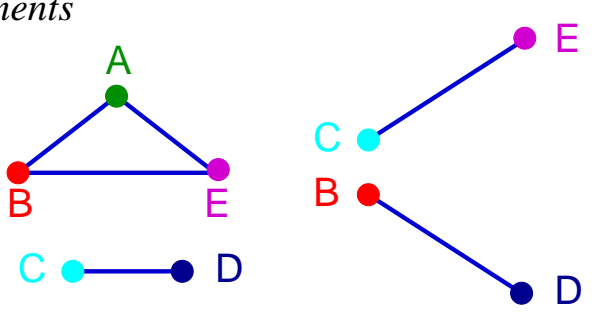

cliques

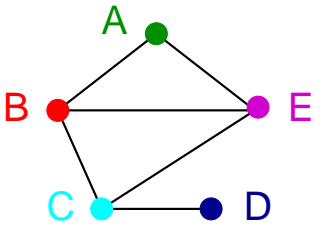

overlap graph

Fig. 5. Decomposition of a cluster of alignments: First the overlap graph $\Gamma$ is computed for a set of alignments. Here we show only a single connected component ("cluster"). The incompatibility graph $\Psi$ summarized pairs of alignments that cannot be derived from a common multiple alignment. Next cliques of its complement $\bar{\Psi}$ are determined. Here we obtain four cliques $C_{1}=\{\mathrm{A}, \mathrm{B}, \mathrm{E}\}, C_{2}=\{\mathrm{C}, \mathrm{D}\}$, $C_{3}=\{\mathrm{C}, \mathrm{E}\}$, and $C_{4}=\{\mathrm{B}, \mathrm{D}\}$. Only $\Gamma\left[C_{1}\right], \Gamma\left[C_{2}\right]$ and $\Gamma\left[C_{3}\right]$ are connected, hence obtain the revised list of cliques $C_{1}, C_{2}, C_{3},\{\mathrm{~B}\},\{\mathrm{D}\}$. Neither of the two isolated points is maximal, i.e., each of them is contained in strictly larger clique, thus the final result of the decomposition are the three non-trivial cliques $C_{1}, C_{2}$, and $C_{3}$.

then

$$
v_{j}^{\prime}=v_{1}+\left(u_{j}^{\prime \prime}-u_{1}^{\prime}\right) \frac{v_{2}-v_{1}}{u_{2}^{\prime}-u_{1}^{\prime}} .
$$

In this way we avoid the explicit construction of the alignments. The correction factor $\left(v_{2}-v_{1}\right) /\left(u_{2}^{\prime}-u_{1}^{\prime}\right)$ is close to 1 if gaps are rare. The inaccuracies incurred by this approximation may lead to slight displacements of the aligned intervals. This can be compensated in the computation by allowing a small tolerance $t$ such that we accept the interval $[a, b] \subseteq[\dot{\subseteq}[c, d]$ iff $a \geq c-t$ and $b \leq d+t$.

Now suppose that somewhere in the search tree we encounter an alignment $A_{k}$ with a trace $\left[p_{1}^{*}, p_{2}^{*}\right]$ at its terminal vertex that is part of the same sequence $p$ as the "root interval" $\left[p_{1}, p_{2}\right]$. If $\left[p_{1}^{*}, p_{2}^{*}\right] \nsubseteq\left[p_{1}, p_{2}\right]$ then at least one sequence interval $\left[u_{1}, u_{2}\right]$ encountered (as trace) somewhere along the path from $\left[p_{1}^{*}, p_{2}^{*}\right]$ to $\left[p_{1}, p_{2}\right]$ would be aligned with two distinct intervals on the same sequence $p$. Consequently, the initial alignment $A_{0}$ and the alignment $A_{k}$ are inconsistent. In this case we do not further extend the search tree from $\left[p_{1}^{*}, p_{2}^{*}\right]$.

We remark that, more abstractly, this procedure can be understood as a depth first search on the path-graph of the overlap graph of the alignments. (The path-graph $P(\Gamma)$ of a graph has as its vertices all paths in $\Gamma$. Two paths are adjacent in $P(\Gamma)$ if one is obtained as an extension by a single edge of 
the other one.) The individual alignments are represented by the paths of length 0 and serve as roots of the search trees. Along each edge of the search tree (i.e., an alignment) we compute the trace (which can be regarded as a vertex label) and check for consistency with the label of the root vertex. For each alignment we therefore obtain a (possibly) empty list of incompatible alignments, and hence the graph $\Psi_{i}$. The Bron-Kerbosch algorithm [7] then produces a non-empty list $\mathcal{C}_{i}=\left\{C_{j}^{i}\right\}$ of cliques. The induced subgraphs $\Gamma_{i}\left[C_{j}^{i}\right]$ are not necessary connected, i.e., they might consist of alignments that do not overlap, Fig. 5 . We thus revise the list of cliques by replacing $\Gamma_{i}\left[C_{j}^{i}\right]$ by all its connected components. It may happen that such a component $C^{\prime}$ is a strict subset of a larger one. In this case $C^{\prime}$ is removed from the list of cliques.

Phylogenetic footprints typically appear in clusters. For the purpose of the analysis in this contribution we pragmatically define a phylogenetic footprint cluster (PFC) as a single consistent clique. In some case one might want to argue that two or several cliques in close proximity should only be counted as a single PFC. For example, in [9] footprints are merged into the same PFC if they are separated by less than 100nt. Since we are interested in relative abundances here this distinction is not important for our conclusions.

\subsection{Multiple Alignments}

The next step is rather straightforward. For each clique $X$ and each sequence $p$ we determine the minimal interval $\left[p^{\prime}, p^{\prime \prime}\right]$ that contains all intervals of $p$ appearing in aligments belonging to $X$. A multiple alignment of these sequence intervals is then produced using a standard program such as clustalw [30] or dialign [24]. So far our data indicate that the final outcome is essentially independent of the multiple alignment algorithm, which at this level serves mostly as a convenient method for visualization.

\subsection{Phylogenetic Distribution of Footprints}

The final processing stage consists of relating the presence/absence pattern of the detected footprints with the established (or assumed) phylogeny of the species in question. Given a phylogenetic tree (in phylip format) as input, tracker automatically compiles an overview table in which clusters are arranged according to common presence/absence patterns together with the parsimony score for the corresponding tree. In addition, overview charts are produced that summarize the locations of the footprints with a common distribution on the phylogenetic tree (not shown here). 


\subsection{Implementation}

The tracker method is implemented as a perl program utilizing ANSI C modules e.g. for determining the inconsistency graph. Furthermore, blastz [28] and clustalw [30] as system calls. The output is provided as a LATEXdocument with included Postscript figures (such as Fig. 2). The tables in the appendix are, appart from the annotation in the last column, taken directly from the tracker output.

\section{A Model for the Amount of Structural Loss of PFCs}

The quantitative data produced by this new algorithm for zebrafish and Takifugu is consistent with the qualitative observation in [9] for zebra fish, namely that there is a massive loss of non-coding sequence conservation associated with cluster duplication. Between 70 and $90 \%$ of the PFCs that are present in shark or human are lost after duplication.

There are three biologically distinct process that can account for this phenomenon: 1) structural, 2) binding site turnover, and 3) adaptive modification. Structural loss is the loss of putative cis-regulatory elements due to gene loss and stochastic resolution of genetic redundancy. Below we will give a more detailed account of what we think can be counted as structural loss. Binding site turnover is loss of noncoding sequence conservation due to the replacement of binding sites even though the function of the enhancer remains conserved. This was first documented in the Drosophila even skipped stripe 2 enhancer [21] and has since been documented for many other invertebrate taxa. In vertebrates, however, no widespread binding site turnover has been documented, which might have to do with a variety of reasons [8]. Adaptive modification would be a change in the sequence of cis-regulatory sites due to directional natural selection and would thus be associated with functional differences.

Loss of non-coding sequence conservation is associated with other structural changes, most notably gene loss. Hence the question arises whether the amount of loss observed is more than expected from the amount of gene loss. To address this question we introduce here a simple model to estimate the amount of PFC loss due to structural changes of the cluster. There are three main sources of PFC loss we consider in this model. Clearly, if a gene is lost, also the associated cis-regulatory elements will be lost, disregarding enhancer sharing. Hence the amount of loss of non-coding sequence conservation has to be calculated in relation to the number of genes which are lost in the focal clusters, in our case the HoxA clusters. We will express these numbers in terms of retention probabilities. 
Table 2

PFC retention statistic after HoxA cluster duplication based on alignment of all seven cluster sequences

\begin{tabular}{|c|c|c|c|c|c|}
\hline Cluster & \#genes & $r(\mathrm{G})$ & \#pPFC & $r(\mathrm{PFC})$ & $r(\mathrm{PFC} \mid \mathrm{G})$ \\
\hline DrHoxAa & 7 & 0.63 & 39 & 0.31 & 0.49 \\
DrHoxAb & 5 & 0.45 & 29 & 0.23 & 0.51 \\
DrHoxA & 12 & 0.55 & 68 & 0.27 & 0.49 \\
\hline TrHoxAa & 9 & 0.82 & 47 & 0.37 & 0.45 \\
TrHoxAb & 5 & 0.45 & 12 & 0.10 & 0.21 \\
TrHoxA & 14 & 0.64 & 59 & 0.23 & 0.37 \\
\hline
\end{tabular}

Dr: zebra fish, Tr: Takifugu \#genes: number of coding genes retained in cluster \#pPFC: number of plesiomorphic phylogenetic footprint cluster, i.e., PFC which have a counterpart in shark or human. See text for the definition of the retention rates.

The total retention probability of an ancestral PFC, $r(\mathrm{PFC})$, depends on the retention probability assuming that the associated coding gene is retained, $r(\mathrm{PFC} \mid \mathrm{G})$, and the probability that the gene is retained $r(\mathrm{G})$,

$$
r(\mathrm{PFC})=r(\mathrm{PFC} \mid \mathrm{G}) r(\mathrm{G}) .
$$

In order to calculate whether the observed rate of $\mathrm{PFC}$ retention is larger than expected for structural reasons one thus has to consider $r(\mathrm{PFC} \mid \mathrm{G})$ rather than $r$ (PFC) directly. We can estimate $r(\mathrm{PFC} \mid \mathrm{G})$ from the observed rate of gene $r(\mathrm{PFC} \mid \mathrm{G})=r(\mathrm{PFC}) / r(\mathrm{G})$. These per gene retention rates are given in Table 2 and are between 0.49 for zebrafish and 0.39 for Takifugu.

There are two other factors we need to take into account in calculating the expected loss of cis-regulatory sequence due to structural changes: the rate of loss due to (1) the loss of cross regulatory interactions among Hox genes and (2) the loss of enhancers due to stochastic resolution of genetic redundancy. The latter plays a role in cases where two paralog genes are retained. It is well known that Hox genes are cross-regulatory, i.e., a Hox gene can be the regulatory input for other Hox genes. It has been observed, both in zebrafish as well as in Takifugu that there are Hox genes that go extinct after cluster duplication, i.e. do not retain a copy of themselves in the duplicated Hox clusters. We assume that with the extinction of that gene its associated enhancer inputs to other Hox genes will be lost as well.

The expected amount of loss due to gene extinction therefore depends on the 
fraction $P\left(\mathrm{G}_{\text {ext }}\right)$ of genes in the whole Hox network that were lost and the fraction $d$ of genes in the Hox network which received regulatory input from these extinct genes. $P\left(\mathrm{G}_{\text {ext }}\right)$ is calculated by counting the number of paralog group members on each of the four clusters in the ancestor of bony fish, i.e. the most recent common ancestor of mouse and zebrafish, for instance. This number is compared with the number of paralog groups which are present in the two duplicated clusters of a teleost.

The number and identity of genes in the most recent common ancestor of bony fish is based on the maximal parsimony reconstruction in [1]. For instance, the ancestor of bony fish has 11 paralog group members in HoxA while zebrafish HoxAa and HoxAb only have a total of 9 paralog groups represented. In other words $18 \%$ (2) of the genes in the ancestral HoxA cluster went extinct in the zebrafish lineage, i.e. have no descendent gene copy in the zebrafish genome. In total there are 42 genes in the four ancestral Hox clusters of which only 37 have at least one descendent genes in zebrafish. This means that $12 \%$ of the genes went extinct, or $P\left(\mathrm{G}_{\text {ext }}\right)=0.12$. Similarly, in the Takifugu Hox clusters there are descendents of 34 of the 42 genes present in the ancestral Hox clusters, which means that the extinction frequency in the Takifugu lineage is $P\left(\mathrm{G}_{\text {ext }}\right)=0.19$ (Chris Amemiya, pers. comm. 2003). The expected rate of PFC loss due to gene extinction is now $d \times P\left(\mathrm{G}_{\text {ext }}\right)$, the corresponding retention probability is therefore $1-d P\left(\mathrm{G}_{\mathrm{ext}}\right)$.

All genes in the Hox cluster arose in some time by gene duplication and are thus all paralogs. There are however different "generations" of paralogs, resulting from different gene and cluster duplication events. We call genes which are related by the most recent gene/cluster duplication 1st order paralogs. The fraction of genes which retain first order paralogs $P\left(1^{\text {st }}\right)$ differs between zebra fish and Takifugu HoxA clusters. There are six genes in zebra fish HoxA clusters which have 1st order paralogs: HoxA-13a/b, HoxA$11 \mathrm{a} / \mathrm{b}$ and HoxA-9a/b. Hence the fraction of 1 st order paralog genes in zebra fish is $P\left(1^{\text {st }}\right)=0.50$. In Takifugu there are ten genes which have first order paralogs retained: HoxA-13a/b, HoxA-11a/b, HoxA-10a/b, HoxA-9a/b, and HoxA-2a/b; hence $P\left(1^{\text {st }}\right)=0.71$. Genes which retain 1 st order paralogs are expected to resolve the genetic redundancy by, on average, losing $50 \%$ of their respective cis-regulatory inputs [12]. Consequently, the larger the fraction of 1st order paralogs the larger the expected amount of PFC loss. If only one copy of the gene survives, by default one would expect that all the relevant cis-regulatory elements are maintained. Hence the probability that a PFC is lost because of stochastic resolution of genetic redundancy is equal to the probability that the associated gene has a 1 st order paralog times $1 / 2$. The 
retention probability of a $\mathrm{PFC}$ is therefore

$$
\begin{aligned}
r_{0} & =\left[\frac{1}{2} P\left(1^{\mathrm{st}}\right)+\left(1-P\left(1^{\mathrm{st}}\right)\right)\right]\left(1-d P\left(\mathrm{G}_{\mathrm{ext}}\right)\right) \\
& =\left(1-\frac{1}{2} P\left(1^{\mathrm{st}}\right)\right)\left(1-d P\left(\mathrm{G}_{\mathrm{ext}}\right)\right)
\end{aligned}
$$

Further, we have to consider a factor for the loss of PFCs due to non-structural causes, such as adaptation or binding site turnover. We call this probability $\alpha$, which means that the retention probability is $(1-\alpha)$. Then the total retention rate of $\mathrm{PFC}$ is

$$
\hat{r}(\mathrm{PFC} \mid \mathrm{G})=r_{0}(1-\alpha)=\left(1-\frac{1}{2} P\left(1^{\mathrm{st}}\right)\right)\left(1-d P\left(\mathrm{G}_{\mathrm{ext}}\right)\right)(1-\alpha)
$$

In this model, we can determine the faction of 1st order paralogs and the gene extinction rate, but we do not know the degree $d$ of cross regulatory connectivity and the rate $\alpha$ of $\mathrm{PFC}$ loss due to non-structural reasons But we have the observed rate of per gene PFC retention and we can thus estimate the degree of non-structural PFC loss $\alpha$, by solving equ.(6) as

$$
\alpha=1-\frac{r(\mathrm{PFC} \mid \mathrm{G})}{\left(1-P\left(1^{\mathrm{st}}\right) / 2\right)\left(1-d P\left(\mathrm{G}_{\mathrm{ext}}\right)\right)}
$$

The only remaining problem is that we do not know $d$. We can at least obtain a lower bound estimate of the rate of non-structural PFC loss, $\hat{\alpha} \leq \alpha$, by assuming $d=1$, i.e., that each Hox gene has a cross regulatory link to every other Hox gene:

$$
\hat{\alpha}=1-\frac{r(\mathrm{PFC} \mid \mathrm{G})}{\left(1-P\left(1^{\mathrm{st}}\right) / 2\right)\left(1-P\left(\mathrm{G}_{\mathrm{ext}}\right)\right)}
$$

In the next section we will apply this model to the analysis of the data from zebrafish and Takifugu Hox-A clusters.

An analogous analysis of the other Hox clusters (Fig. 2) is difficult at present since the sequences for Takifugu and zebrafish are incomplete and/or the corresponding outgroup sequences are not yet available.

The preliminary PFC statistics for the Hox-B clusters are compiled in Table 3. These numbers should be viewed with caution. In particular, the PFC retention rates $r(\mathrm{PFC})$ are upper bounds since we miss PFCs that have been lost completely in either mamalia or fish lineages. The quality of these data will improve when further outgroups, e.g., the B-cluster of bichir, become available. An additional source of uncertainty is the fact that the 3'-end of the $\mathrm{DrBb}$ cluster is missing in the currently available assembly, see Fig. 2. 
Table 3

PFC retention statistic after HoxB cluster duplication based on alignment of all seven cluster sequences. Note that due to limited data the retentation rates are only upper bounds. For the DrHoxBa cluster we count only the genes that are contained in available sequence data, see the caption of Fig. 2 for details.

\begin{tabular}{|c|c|c|c|c|c|}
\hline Cluster & \#genes & $r(\mathrm{G})$ & \#pPFC & $r(\mathrm{PFC})$ & $r(\mathrm{PFC} \mid \mathrm{G})$ \\
\hline DrHoxBa & $8+$ & $0.8+$ & 62 & 0.53 & $<0.66$ \\
DrHoxBb & 4 & 0.4 & 43 & 0.37 & 0.92 \\
DrHoxB & $12+$ & $0.6+$ & 105 & 0.45 & $<0.75$ \\
\hline TrHoxBa & 8 & 0.8 & 69 & 0.59 & 0.74 \\
TrHoxBb & 3 & 0.3 & 35 & 0.30 & 1.00 \\
TrHoxB & 11 & 0.55 & 104 & 0.44 & 0.8 \\
\hline
\end{tabular}

For the Hox-C and Hox-D clusters sequence data of duplicate clusters are currently not publicly available with sufficient data quality.

\section{Results}

There are 126 PFC that are found in either the shark or human HoxA cluster or both. In contrast, there are only 68 of those retained in at least one zebrafish clusters and 59 are retained in at least one Takifugu HoxA cluster, while only 8 and 9 PFCs, resp., survived in both paralog clusters. This corresponds to a retention rate of $27 \%$ and $23 \%$ respectively (Table 2 ). This confirms the qualitative observation in [9], that Hox cluster duplication is associated with a massive loss of non-coding sequence conservation. In this section we will use the model proposed above to set this rate of sequence conservation loss in relation to gene loss. But before we go into the analysis of the data we want to point out a methodological issue in scoring the rate of PFC loss in this type of data.

There are 53 PFCs in zebrafish and Takifugu that have no counterpart in shark or human; of these 14 were found only in zebrafish and 10 only in Takifugu. These PFCs most likely correspond either to cis-regulatory elements which were lost independently in the shark and human lineage or which are PFCs acquired in the stem lineage of teleost fish. These PFCs, however, cannot be used to estimate the rate of PFC retention after cluster duplication, because one can not detect the PFCs that have only been maintained in one of the paralog clusters. For that reasons we ignore the number of PFCs which have 
Table 4

Conditional PFC retention statistic after HoxA cluster duplication based on the predictions of the structural loss model. Note that the predicted retention rate based on the structural loss model is consistently higher than observed rate of loss, indicating other, non-structural causes of sequence conservation loss. There is a notable asymmetry in the predicted minimal rate of non-structural conservation loss between the clusters. In zebrafish the HoxAa cluster seems to be twice as strongly modified while in Takifugu the HoxAb cluster has an exceptionally high minimal modification rate of 0.46 . This pattern is consistent with rates of coding sequence evolution among paralog Hox genes in these species (Takahashi et al., in prep.).

\begin{tabular}{|c|c|c|c|c|c|}
\hline Cluster & \#genes & $P\left(1^{\text {st }}\right)$ & \multicolumn{2}{|c|}{$r(\mathrm{PFC} \mid \mathrm{G})$} & $\hat{\alpha}$ \\
& & & data & equ. $(5)$ & \\
\hline DrHoxAa & 7 & 0.43 & 0.49 & 0.69 & 0.29 \\
DrHoxAb & 5 & 0.60 & 0.51 & 0.62 & 0.18 \\
DrHoxA & 12 & 0.50 & 0.49 & 0.66 & 0.26 \\
\hline TrHoxAa & 9 & 0.56 & 0.45 & 0.58 & 0.22 \\
TrHoxAb & 5 & 1.00 & 0.21 & 0.40 & 0.48 \\
TrHoxA & 14 & 0.71 & 0.37 & 0.52 & 0.29 \\
\hline
\end{tabular}

no counterpart in shark or human. We have to keep in mind that the counts of PFCs are just a sample of all putative cis-regulatory elements involved. If, however, the retention rates of these PFCs are comparable to those present in shark and human, the statistics will still give valid estimates.

In order to account for the loss of genes in the focal HoxA clusters after duplication, we calculate the conditional retention rate, see above. The conditional retention rate is about $50 \%$ for zebrafish and $37 \%$ overall for Takifugu. This suggests that, corrected for gene loss in the HoxA cluster, Takifugu has a lower retention rate than zebrafish. The two paralog clusters in Takifugu have a strongly different retention rates, 0.21 for the HoxAb cluster and 0.45 for HoxAa cluster. In contrast, the conditional retention rate in zebrafish is about the same for both clusters, 0.49 and 0.51 respectively.

Applying our model for the structural loss of non-coding sequence conservation to the PFC data of the Hox-A clusters shows that the observed amount of retention is in all cases less than predicted as the minimal amount of retention if only structural reasons would cause loss of sequence conservation. Hence the model is consistent with the data, in the sense that we do not observe more conservation than the minimal amount predicted by this model.

Calculating the minimal probability of PFC loss, due to non-structural reasons 
(binding site turnover and directional selection) shows that in zebrafish and Takifugu this rate is roughly comparable, about $26 \%$ and $29 \%$ respectively, see Table 4 . The slightly higher rate in Takifugu, however, is entirely accounted for by the higher rate estimate for the HoxAb cluster. The non-structural modification rate in the HoxAa cluster is 0.22 , about the same as in zebrafish, while the minimal rate of non-structural modification in the Takifugu HoxAb cluster is $48 \%$. This suggests that there was a differential loss of non-coding sequence conservation in the Takifugu HoxAb cluster. Assuming that the probability of functionally conservative binding site turnover is about the same in the two paralog clusters, this result strongly suggests that the Takifugu HoxAb cluster experienced adaptive modification at a higher rate than both the Takifugu HoxAa cluster and either of the zebrafish clusters.

\section{Discussion}

The evolution of development is to a large part based on changes in the cisregulatory elements of developmental genes [10]. Hence the evolutionary genetics of development requires tools for analyzing the rate and pattern of evolution of cis-regulatory sequences. This task is more difficult than the study of coding sequence evolution, because we lack a "genetic code" for the interpretation of non-coding DNA sequences. There are two approaches used in the current literature. One requires a model species for which the cis-regulatory sequences have been characterized experimentally and where the upstream factors are known. This approach provides the highest level of detail but is limited to a few well characterized genes. The other method was pioneered by Greg Wray and collaborators [14] and looks for the statistical over or under representation of known binding sites. For instance, Wray has shown that overall known binding sites are on average less frequent than expected on the basis of nucleotide frequencies in prokaryote genomes [14].

The novel computational method presented in this paper opens up an alternative avenue to the study of non-coding sequence evolution. It uses the fact that, at least in vertebrates, cis-regulatory sites have been shown to evolve at a lower rate than surrounding sequences. The software tool presented here allows the identification of partially conserved, homologous sequences in many long sequences. With a sufficient number of sequences from phylogenetically well placed taxa it is then possible to study the origin, maintenance and loss of conserved sequence segments among different lineages. The method, which is based on pairwise sequence comparisons and subsequent assembly and filtering steps, is designed to deal with a moderately large number of (very) long sequences. The survey of the seven Hox-A clusters reported here, for instance, requires less than $5 \mathrm{~min}$ on a modern PC. The tracker tool can therefore be used for much larger datasets as the resource usage scales approximately as 
$\mathcal{O}\left(L \times N^{2}\right)$ for $N$ input sequences of length $L$.

Here we have applied this tool to the modifications of non-coding sequences following Hox cluster duplication in teleosts, zebrafish and Takifugu. In principle this method can also be used to study the cis-regulatory changes associated with other evolutionary changes. For instance, it is known that the AbdB related HoxD cluster genes acquired a novel pattern of regulation with the origin of the tetrapod limb $[25,33]$. It should be possible to detect differences in the pattern of cis-regulatory sequence conservation between basal fishes and tetrapods. However, no data from appropriately placed taxa is currently available.

The comparative analysis of sequences is much aided by models of sequence evolution. In the case of coding sequences a large number of models can be used to detect unusual patterns of sequence change [13]. No comparable models are available for the analysis of non-coding sequence. In this paper we have proposed a simple model for the loss of non-coding sequence conservation after gene and cluster duplication. The purpose of this model is to estimate the amount of PFC loss that can be attributed to "structural" reasons, such as gene loss. The results show that the observed amount of non-coding sequence modification is in all cases higher than expected solely for structural reasons. It is hard to distinguish between the two possible reasons for this excess in the loss of sequence conservation: binding site turnover and adaptive modification. The former changes sequences of cis-regulatory elements without affecting function, while the latter is the cis-regulatory trace of changes in the function of the associated genes. It is hard to distinguish between these two factors contributing to the non-structural loss of sequence conservation. In the data set analyzed in this paper, however, we found a possible signature of adaptive loss of sequence conservation. In Takifugu the rate of non-structural sequence modification is twice as high in the HoxAb cluster than in the HoxAa cluster (see Table 4). Since there is no reason to assume that the rate of binding site turnover should be different between paralog Hox clusters, the most parsimonious interpretation is that, in Takifugu, the HoxAb cluster experienced a higher amount of adaptive change in its cis-regulatory elements than the HoxAa cluster. This suggestion can be tested by expression studies and transgenic tests of non-coding sequences.

\section{Acknowledgments}

Helpful discussion with Frank Ruddle, Türker Bıyıoğlu, Chi-hua Chiu, Ivo L. Hofacker, Kazuhiko Takahashi, information on the Takifugu gene complement by Chris Amemiya, and computational assistence in the early stages of this project by Paul Perco are gratefully acknowledged. 


\section{References}

[1] A. Amores, A. Force, Y. L. Yan, L. Joly, C. Amemiya, A. Fritz, R. K. Ho, J. Langeland, V. Prince, Y. L. Wang, M. Westerfield, M. Ekker, and J. H. Postlethwait. Zebrafish hox clusters and vertebrate genome evolution. Science, 282:1711-1714, 1998.

[2] S. Aparicio, J. Chapman, E. Stupka, N. Putnam, J.-m. Chia, P. Dehal, A. Christoffels, S. Rash, S. Hoon, A. Smit, M. D. S. Gelpke, J. Roach, T. Oh, I. Y. Ho, M. Wong, C. Detter, F. Verhoef, P. Predki, A. Tay, S. Lucas, P. Richardson, S. F. Smith, M. S. Clark, Y. J. K. Edwards, N. Dogget, A. Zharkikh, S. V. Tavtigian, D. Pruss, M. Barstead, C. Evans, H. Baden, J. Powell, G. Glusman, L. Rowen, L. Hood, T. Y. H., G. Elgar, T. Hawkins, B. Venkatesh, D. Rokhsar, and S. Brenner. Whole-genome shotgun assembly and analysis of the genome of Fugu rubripes. Science, 297:1301-1310, 2002.

[3] S. Aparicio, K. Hawker, A. Cottage, Y. Mikawa, L. Zuo, B. Venkatesh, E. Chen, R. Krumlauf, and S. Brenner. Organization of the Fugu rubripes Hox clusters: evidence for continuing evolution of vertebrate Hox complexes. Nat. Genetics, 16:79-83, 1997.

[4] M. I. Arnone and E. H. Davidson. The hardwiring of development: Organization and function of genomic regulatory systems. Development, 124:1851-1864, 1997.

[5] M. Blanchette, B. Schwikowski, and M. Tompa. Algorithms for phylogenetic footprinting. J. Comp. Biol., 9:211-223, 2002.

[6] M. Blanchette and M. Tompa. Discovery of regulatory elements by a computational method for phylogenetic footprinting. Genome Research, 12:739$748,2002$.

[7] C. Bron and J. Kerbosch. Algorithm 457: Finding all cliques of an undirected graph. CACM, 16:575-577, 1973.

[8] A. J. Carter and G. P. Wagner. Evolution of functionally conserved enhancers can be accelerated in large populations: a population-genetic model. Proc. $R$. Soc. Lond. B Biol. Sci., 269:953-960, 2002.

[9] C.-h. Chiu, C. Amemiya, K. Dewar, C.-B. Kim, F. H. Ruddle, and G. P. Wagner. Molecular evolution of the HoxA cluster in the three major gnathostome lineages. Proc. Natl. Acad. Sci. USA, 99:5492-5497, 2002.

[10] E. H. Davidson, J. P. Rast, P. Oliveri, A. Ransick, C. Calestani, C.-H. Yuh, T. Minokawa, G. Amore, V. Hinman, C. Arenas-Mena, O. Otim, C. T. Brown, C. B. Livi, P. Y. Lee, R. Revilla, A. G. Rust, Z. jun Pan, M. J. Schilstra, P. J. C. Clarke, M. I. Arnone, L. Rowen, R. A. Cameron, D. R. McClay, L. Hood, and H. Bolouri. A genomic regulatory network for development. Science, 295:16691678, 2002.

[11] L. Duret and P. Bucher. Searching for regulatory elements in human noncoding sequences. Curr. Opin. Struct. Biol., 7:399-406, 1997. 
[12] A. Force, M. Lynch, F. B. Pickett, A. Amores, Y.-1. Yan, and J. Postlethwait. Preservation of duplicate genes by complementary, degenerative mutations. Genetics, 151:1531-1545, 1999.

[13] D. Graur and W.-H. Li. Fundamentals of Molecular Evolution. Sinauer Associates, Sunderland, Massachusetts, 2000.

[14] M. W. Hahn, J. E. Stajich, and G. A. Wray. Selection against spurious transcription factor binding sites shapes genomes. Mol. Biol. Evol., 2003. in press.

[15] G. Hertz and G. D. Stormo. Identifying DNA and protein patterns with statistically significant alignments of multiple sequences. Bioinformatics, 15:563-577, 1999.

[16] J. D. Hughes, P. W. Estep, S. Tavazoie, and G. M. Church. Computational identification of cis-regulatory elements associated with groups of functionally related genes in saccharomyces cerevisiae. J. Mol. Biol., 296:1205-1214, 2000.

[17] M. Kimura. The Neutral Theory of Molecular Evolution. Cambridge University Press, Cambridge, UK, 1983.

[18] J. Y. Leung, F. E. McKenzie, A. M. Uglialoro, P. O. Flores-Villanueva, B. C. Sorkin, E. J. Yunis, D. L. Hartl, and A. E. Goldfeld. Identification of phylogenetic footprints in primate tumor necrosis factor- $\alpha$ promoters. Proc. Natl. Acad. Sci. USA, 97:6614-6618, 2000.

[19] G. Loots, I. Ovcharenko, L. Pachter, I. Dubchak, and R. E. rVISTA for comparative sequence-based discovery of functional transcription factor binding sites. Genome. Res., 12:832-839, 2002.

[20] M. Z. Ludwig. Functional evolution of noncoding DNA. Curr. Op. Genet. Devel., 12:634-639, 2002.

[21] M. Z. Ludwig, C. Bergman, N. H. Patel, and M. Kreitman. Evidence for stabilizing selection in a eukaryotic enhancer element. Nature, 403:564-567, 2000 .

[22] E. Málaga-Trillo and A. Meyer. Genome duplications and accelerated evolution of Hox genes and cluster architecture in teleost fishes. Amer. Zool., 41:676-686, 2001.

[23] J. Manen, V. Savolainen, and P. Simon. The $a t p B$ and $r b c L$ promoters in plastid DNAs of a wide dicot range. J. Mol. Evol., 38:577-582, 1994.

[24] B. Morgenstern. DIALIGN 2: improvement of the segment-to-segment approach to multiple sequence alignment. Bioinformatics, 15:211-218, 1999.

[25] C. E. Nelson, B. A. Morgan, A. C. Burke, E. Laufer, E. DiMambro, L. C. Murtaugh, E. L. Gonzales, Tessasollo, L. Parada, and T. C. Analysis of Hox gene expression in the chick limb bud. Development, 122:1449-1466, 1996. 
[26] V. E. Prince. The hox paradox: More complex(es) than imagined. Developmental Biology, 249:1-15, 2002.

[27] F. P. Roth, J. D. Hughes, P. W. Estep, and G. M. Church. Finding DNA regulatory motifs within unaligned noncoding sequences clustered by wholegenome mRNA quantitation. Nat. Biotechnol., 16:939-945, 1998.

[28] S. Schwartz, Z. Zhang, K. A. Frazer, A. Smit, C. Riemer, J. Bouck, R. Gibbs, R. Hardison, , and W. Miller. PipMaker - a web server for aligning two genomic dna sequences. Genome Research, 4:577-586, 2000.

[29] D. A. Tagle, B. F. Koop, M. Goodman, J. L. Slightom, D. L. Hess, and R. T. Jones. Embryonic epsilon and gamma globin genes of a prosimian primate (galago crassicaudatus). Nucleotide and amino acid sequences, developmental regulation and phylogenetic footprints. J. Mol. Biol., 203:439-455, 1988.

[30] J. D. Thompson, D. G. Higgs, and T. J. Gibson. CLUSTALW: improving the sensitivity of progressive multiple sequence alignment through sequence weighting, position specific gap penalties, and weight matrix choice. Nucl. Acids Res., 22:4673-4680, 1994.

[31] A. Wagner. Genes regulated cooperatively by one or more transcription factors and their identification in whole eukaryotic genomes. Bioinformatics, 15:776$784,1999$.

[32] C. T. Workman and G. D. Stormo. ANN-Spec: a method for discovering transcription factor binding sites with improved specificity. In Pacific Symposium on Biocomputing, pages 467-78, 2000.

[33] J. Zákány and D. Duboule. Hox genes in digit development and evolution. Cell Tissue Res., 296:19-25, 1999.

[34] Fugu genome database, 2002. version 2.0: http://genome.jgi-psf .org/fugu3/fugu3.home.html, version 3.0: http://genome.jgi-psf .org/fugu6/fugu6.home.html.

[35] The Danio rerio sequencing project, 2002.

http://www. sanger.ac.uk/Projects/Drerio/. 


\section{Appendix A: Tables}

The analysis of the Hox-A clusters was performed in two steps. A re-evaluation of the analysis reported in [9], see Table 5ff, and a combined evaluation that uses the sequences from Takifugu as well. Tables $12 \mathrm{ff}$ summarize the additional footprints and has been used as the basis for the summary statistics reported in Table 2.

The tracker program recovers all footprints reported in [9] with the three exceptions, included in italics in Tables 5ff, briefly discussed below. We find that tracker is more sensitive, detecting about three times as many hits, some of which, however, are combined into the same PFC in [9].

11-9-b is a footprint of length 9. It is too short to be accepted as significant hit with the default parameter settings of tracker:

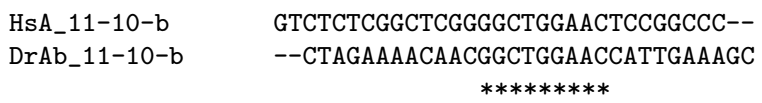

up13-c does not exist at the reported location. A clustalw alignment yields

HfM_up13-c

DrHoxAa_up13-c

ACAGAAAACAGTTTTTGTAAAATAGTCATTTAGTATTAAAT

CAAAAAAAAAAAAAAACACTG---

$* * * * * * * *$

5-4-b does not correspond to a significant match at the reported positions. The corresponding clustalw alignment is

HsA_5-4-b

HfM_5-4-b

HsA_5-4-b

HfM_5-4-b

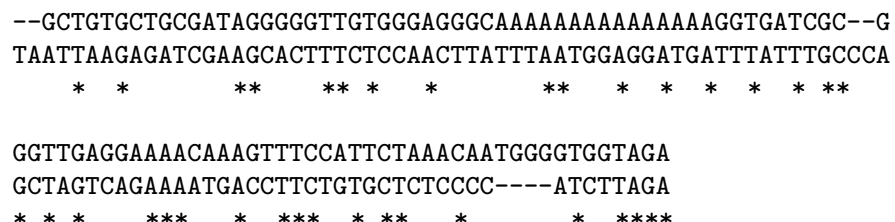


Table 5. Comparison with [9].

The last column gives the designation of the footprints from [9]. Footprints that were not found by tracker are listed in italics without numbering, + denotes novel ones. +XXX means that we found footprint also in XXX; analogously, -XXX means that the footprint was detected in the Hox cluster XXX in the previous study [9] but was not found in this sequence by the tracker program with the default parameter setting. Positions of footprints that are missing in some sequences in the tracker output are given in parentheses. Differences between the published position numbers of the DrAa sequence and our data are explained by the use of two versions of the DrAa sequence in [9].

\begin{tabular}{|c|c|c|c|c|c|c|c|c|c|c|}
\hline Footprint & $\mathrm{Hf}$ & & $\mathrm{H}$ & & DrAc & & DrAl & & $\mathrm{MsA}$ & PFC 5'-3' \\
\hline 1 & 865 & 23 & & & & & 1553 & 23 & & + \\
\hline 2 & 2891 & 51 & & & & & 39450 & 51 & & + \\
\hline 3 & & & 8197 & 31 & & & 33525 & 31 & & + \\
\hline 4 & & & & & 2283 & 76 & 7560 & 76 & & + \\
\hline 5 & & & & & 2287 & 70 & 6101 & 70 & & + \\
\hline 6 & & & & & 3246 & 62 & 29283 & 60 & & + \\
\hline 7 & & & & & 7147 & 46 & 32044 & 46 & & + \\
\hline 8 & & & 13150 & 59 & 15129 & 59 & & & & + \\
\hline 9 & & & 13216 & 30 & 15198 & 31 & & & & + \\
\hline 10 & & & 13258 & 12 & 15241 & 12 & & & & + \\
\hline 11 & & & 15102 & 41 & & & 7692 & 47 & & + \\
\hline 12 & 3734 & 81 & 20391 & 84 & & & & & & + \\
\hline 13 & 3881 & 23 & & & 4203 & 23 & & & & + \\
\hline 14 & & & 25741 & 38 & & & 15607 & 33 & & + \\
\hline 15 & & & 27295 & 29 & & & 35475 & 29 & & + \\
\hline 16 & 5901 & 75 & 28483 & 75 & & & & & & + \\
\hline 17 & 5949 & 23 & 4134 & 23 & & & & & & + \\
\hline 18 & 6483 & 120 & 45120 & 121 & & & & & & upstream of 13 -a \\
\hline 19 & 6775 & 40 & 45433 & 37 & & & & & & upstream of $13-b$ \\
\hline & 8558 & 40 & & & 21743 & 19 & & & & upstream of 13-c \\
\hline 20 & 11868 & 26 & & & & & 47489 & 26 & & + \\
\hline
\end{tabular}


Table 6. Table 5 continued.

\begin{tabular}{|c|c|c|c|c|c|c|c|c|c|c|}
\hline Footprint & $\mathrm{HfI}$ & & $\mathrm{HsA}$ & & $\overline{\text { DrA }}$ & & DrAl & & MsA & PFC 5'-3' \\
\hline 21 & & & & & 16307 & 78 & 22716 & 78 & & + \\
\hline 22 & & & & & 18316 & 42 & 29824 & 42 & & + \\
\hline 23 & & & & & 18387 & 55 & 29928 & 55 & & + \\
\hline 24 & 13192 & 120 & 53810 & 88 & 22652 & 65 & 58295 & 121 & & upstream of $13-\mathrm{d}$ \\
\hline 25 & 13360 & 13 & & & & & 58469 & 14 & & + \\
\hline 26 & 16127 & 49 & & & & & 58996 & 48 & & 13pp -DrAa + DrAb \\
\hline 27 & 16233 & 57 & & & & & 59103 & 56 & & 13pp -DrAa + DrAb \\
\hline 28 & 19133 & 112 & 59505 & 114 & 25574 & 24 & & & & 13-11-a +DrAa \\
\hline 29 & 20828 & 47 & & & & & 63519 & 47 & & + \\
\hline 30 & 27207 & 32 & & & 28565 & 32 & & & & + \\
\hline 31 & 27545 & 30 & & & & & 66363 & 30 & & + \\
\hline 32 & 27606 & 116 & 68103 & 118 & & & & & & + \\
\hline 33 & & & 70181 & 58 & 28402 & 58 & & & & + \\
\hline 34 & & & & & 29483 & 35 & 67002 & 35 & & + \\
\hline 35 & 29781 & 168 & 70665 & 152 & 31068 & 118 & 67981 & 132 & & 13-11pp \\
\hline 36 & & & & & 33896 & 155 & 71142 & 153 & & 11-9-a DrAa(29667) \\
\hline 37 & 34076 & 42 & & & 43022 & 42 & & & & + \\
\hline 38 & 34423 & 77 & 75337 & 78 & & & & & & $11-10-\mathrm{a}$ \\
\hline & & & 76034 & 9 & & & 71322 & 9 & & $11-10-b$ \\
\hline 39 & 35043 & 77 & 76069 & 52 & 34212 & 31 & 71442 & 74 & & $11-10-c+\operatorname{Dr} A$ \\
\hline 40 & 41272 & 55 & & & & & 71853 & 55 & & + \\
\hline 41 & & & 78189 & 21 & 32835 & 21 & & & & + \\
\hline 42 & 43143 & 93 & 81631 & 94 & & & 73488 & 75 & & + \\
\hline
\end{tabular}


Table 7. Table 5 continued.

\begin{tabular}{|c|c|c|c|c|c|c|c|c|c|c|c|}
\hline Footprint & HfM & & $\mathrm{Hs}$ & & DrA & & DrA & & Ms & & PFC 5'-3' \\
\hline 43 & 46400 & 43 & 85314 & 39 & & & & & & & $10-9-\mathrm{a}$ \\
\hline 44 & 46546 & 24 & 85435 & 24 & & & & & & & $10-9-\mathrm{a}$ \\
\hline 45 & 46591 & 188 & 85479 & 187 & & & & & 2977 & 139 & $10-9-\mathrm{a}$ \\
\hline 46 & 47542 & 116 & 86410 & 116 & 41556 & 97 & 76755 & 93 & 3393 & 97 & 10-9-b DrAa(37297) \\
\hline 47 & & & & & & & 76892 & 16 & 3556 & 16 & + \\
\hline 48 & 48333 & 30 & 87347 & 38 & 41872 & 35 & 77048 & 44 & 3791 & 49 & $10-9-\mathrm{c}+\mathrm{HsA}+\mathrm{DrAa}+\mathrm{MsA}$ \\
\hline 49 & 52969 & 35 & 90122 & 35 & & & & & & & $10-9-\mathrm{d}$ \\
\hline 50 & 53030 & 45 & 90215 & 44 & & & & & & & + \\
\hline 51 & 53084 & 55 & 90267 & 55 & & & & & & & 10-9pp -MsA(6219) \\
\hline 52 & 53229 & 28 & 90412 & 28 & & & & & & & 10-9pp -MsA \\
\hline 53 & 53264 & 42 & 90452 & 41 & & & & & & & $10-9 \mathrm{pp}-\mathrm{MsA}$ \\
\hline 54 & & & & & 43987 & 63 & & & 6298 & 64 & + \\
\hline 55 & & & & & 45766 & 47 & & & 8387 & 46 & + \\
\hline 56 & & & & & & & 77140 & 16 & 3893 & 16 & + \\
\hline 57 & & & & & & & 77166 & 94 & 3929 & 96 & + \\
\hline 58 & 56953 & 99 & 94192 & 61 & 46679 & 175 & 81365 & 81 & 8912 & 182 & 9-7-a +DrAa +Drab +MsA \\
\hline 59 & 57228 & 219 & 94465 & 223 & 47016 & 208 & & & 9511 & 229 & 9-7-b + DrA + MsA \\
\hline 60 & 57682 & 31 & 94836 & 31 & & & & & & & + \\
\hline 61 & 59503 & 39 & & & & & 87245 & 36 & & & + \\
\hline 62 & & & 97345 & 38 & & & & & 9394 & 38 & + \\
\hline 63 & 62154 & 12 & 99257 & 12 & & & & & & & 9-7-pp \\
\hline 64 & 62176 & 33 & 99279 & 32 & & & & & 11485 & 29 & 9-7-pp \\
\hline
\end{tabular}


Table 8. Table 5 continued.

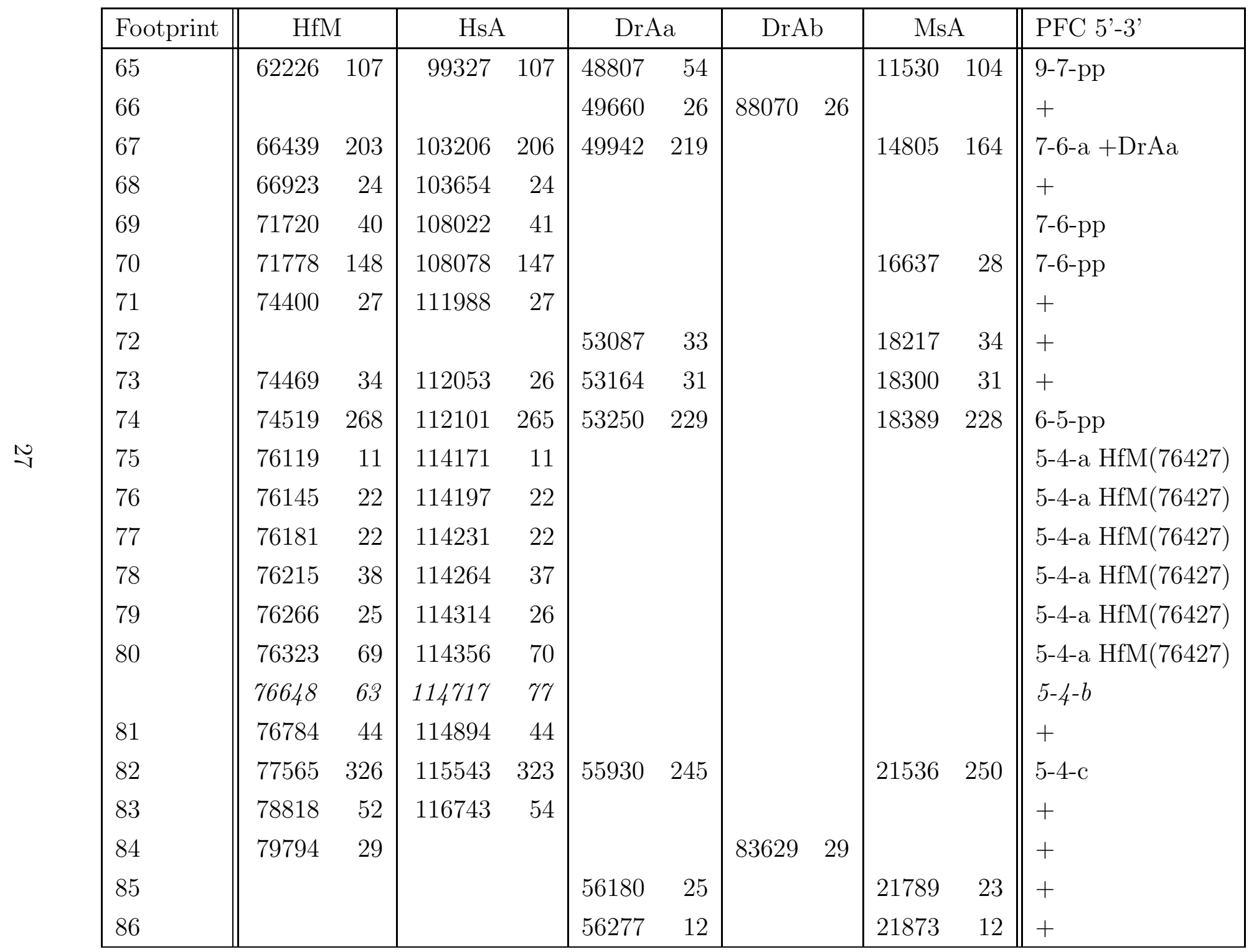


Table 9. Table 5 continued.

\begin{tabular}{|c|c|c|c|c|c|c|c|c|c|c|c|}
\hline Footprint & $\mathrm{Hfl}$ & & $\mathrm{HsA}$ & & DrA & & DrAl & & $\mathrm{MsA}$ & & PFC 5'-3' \\
\hline 87 & 81947 & 71 & 119346 & 105 & 57520 & 105 & & & 23483 & 104 & $5-4-d+D r A a$ \\
\hline 88 & 82035 & 16 & & & & & & & 23604 & & + \\
\hline 89 & 82436 & 286 & 119799 & 284 & 57972 & 163 & & & 24139 & 180 & $5-4-\mathrm{e}+\mathrm{DrAa}$ \\
\hline 90 & 82749 & 16 & 120098 & 15 & & & & & & & + \\
\hline 91 & & & & & 58177 & 68 & & & 24365 & 70 & + \\
\hline 92 & 84826 & 231 & 121990 & 231 & 59802 & 175 & & & 27247 & 180 & $5-4-\mathrm{f}+\mathrm{MsA}$ \\
\hline 93 & & & 122238 & 27 & & & 86591 & 27 & & & + \\
\hline 94 & 85596 & 41 & 122775 & 40 & & & 88770 & 23 & & & + \\
\hline 95 & 85651 & 41 & 122822 & 41 & & & & & & & $5-4-g$ \\
\hline 96 & 85787 & 19 & & & & & 85007 & 19 & & & + \\
\hline 97 & 85814 & 29 & & & & & 85029 & 31 & & & + \\
\hline 98 & 87745 & 114 & 125173 & 76 & 61442 & 176 & & & 28922 & 183 & + \\
\hline 99 & 91064 & 132 & 128822 & 129 & & & & & & & $4-3-\mathrm{a}$ \\
\hline 100 & 91515 & 58 & 129461 & 58 & & & & & & & + \\
\hline 101 & 91602 & 30 & 129556 & 30 & & & & & & & + \\
\hline 102 & 92853 & 91 & 131248 & 89 & & & & & & & + \\
\hline 103 & 93227 & 73 & 131592 & 77 & & & & & & & + \\
\hline 104 & 93311 & 42 & 131680 & 42 & & & & & & & + \\
\hline 105 & 93372 & 81 & 131766 & 83 & & & & & & & + \\
\hline 106 & 94873 & 34 & & & & & 88361 & 34 & & & + \\
\hline 107 & 98246 & 55 & 136897 & 58 & & & & & & & + \\
\hline 108 & 98424 & 35 & 137066 & 37 & & & & & & & + \\
\hline
\end{tabular}


Table 10. Table 5 continued.

\begin{tabular}{|c|c|c|c|c|c|c|c|c|c|c|}
\hline Footprint & $\overline{\mathrm{HfM}}$ & & $\overline{\mathrm{HsA}}$ & & $\overline{\text { DrA }}$ & & $\overline{D r A b}$ & & $\mathrm{MsA}$ & PFC 5'-3' \\
\hline 109 & 98476 & & 137119 & 58 & & & & & & + \\
\hline 110 & 98868 & 148 & 137526 & 147 & & & & & & + \\
\hline 111 & & & & & 65895 & 19 & 87490 & 19 & & + \\
\hline 112 & 99108 & 85 & 137815 & 82 & 67086 & 81 & & & & + \\
\hline 113 & 99764 & 29 & & & & & 89449 & 29 & & + \\
\hline 114 & 101931 & 276 & 140542 & 277 & & & & & & + \\
\hline 115 & 102590 & 50 & & & 69681 & 56 & & & & + \\
\hline 116 & 102694 & 27 & 141968 & 27 & & & & & & + \\
\hline 117 & 102966 & 86 & 142331 & 46 & 70109 & 86 & & & & $4-3-b$ \\
\hline 118 & 103058 & 129 & 142393 & 129 & 70220 & 50 & & & & + \\
\hline 119 & 105041 & 154 & 144063 & 157 & & & & & & + \\
\hline 120 & 105199 & 33 & 144236 & 32 & & & & & & + \\
\hline 121 & 106120 & 92 & 145095 & 94 & 71542 & 39 & & & & 4-3-pp + HsA \\
\hline 122 & 106233 & 124 & 145205 & 135 & 71593 & 132 & & & & $4-3-\mathrm{pp}+\mathrm{HsA}$ \\
\hline 123 & 109890 & 95 & 148351 & 96 & & & & & & + \\
\hline 124 & 109999 & 217 & 148482 & 218 & & & & & & + \\
\hline 125 & & & 151198 & 30 & & & 89631 & 28 & & + \\
\hline 126 & & & & & 73712 & 35 & 87719 & 35 & & + \\
\hline 127 & 112888 & 123 & 151235 & 121 & 75190 & 114 & 89669 & 117 & & + \\
\hline 128 & 113671 & 123 & 152783 & 127 & & & & & & $3-2-a$ \\
\hline 129 & 113939 & 243 & 153130 & 247 & & & 90535 & 218 & & 3-2-pp \\
\hline
\end{tabular}


Table 11. Table 5 continued.

\begin{tabular}{|c|c|c|c|c|c|c|c|c|c|}
\hline \multirow{2}{*}{\begin{tabular}{|l|} 
Footprint \\
130
\end{tabular}} & \multicolumn{2}{|l|}{ HfM } & \multicolumn{2}{|l|}{$\mathrm{HsA}$} & \multicolumn{2}{|l|}{ DrAa } & \multirow[t]{2}{*}{ DrAb } & \multirow[t]{2}{*}{ MsA } & PFC 5'-3' \\
\hline & 116088 & 86 & 155551 & 83 & \multirow{9}{*}{\multicolumn{2}{|c|}{$92267 \quad 39$}} & & & + \\
\hline 131 & 116229 & 30 & 155683 & 30 & & & & & + \\
\hline 132 & 116301 & 11 & 155747 & 11 & & & & & + \\
\hline 133 & 117348 & 99 & 156872 & 100 & & & & & $2-1-\mathrm{a}$ \\
\hline 134 & 117460 & 78 & 156985 & 79 & & & & & $2-1-\mathrm{a}$ \\
\hline 135 & 119953 & 54 & 159818 & 54 & & & & & + \\
\hline 136 & 120009 & 44 & 159883 & 44 & & & & & + \\
\hline 137 & 120063 & 69 & 159973 & 72 & & & & & + \\
\hline 138 & & & 161549 & 39 & & & & & + \\
\hline 139 & 121736 & 18 & 161979 & 16 & & & & & + \\
\hline 140 & 121808 & 11 & 162032 & 11 & & & & & + \\
\hline 141 & 121838 & 56 & 162050 & 57 & & & & & + \\
\hline 142 & 122218 & 85 & 162406 & 90 & & & & & + \\
\hline 143 & 122334 & 39 & 162528 & 39 & & & & & + \\
\hline 144 & 122397 & 12 & 162592 & 12 & & & & & + \\
\hline 145 & 122423 & 25 & 162618 & 23 & & & & & + \\
\hline 146 & 122483 & 17 & 162663 & 17 & & & & & + \\
\hline 147 & & & 162790 & 27 & 113979 & 27 & & & + \\
\hline 148 & 122765 & 79 & 162923 & 79 & & & & & + \\
\hline
\end{tabular}


Table 12. Footprints in Takifugu rubripes.

Data from Table 5 that do not involve a Takifugu rubripes match are not listed. Clusters that are separated into more than one entry are sometimes merged into a single cluster here. Cluster numbers in brackets refer to Table 5.

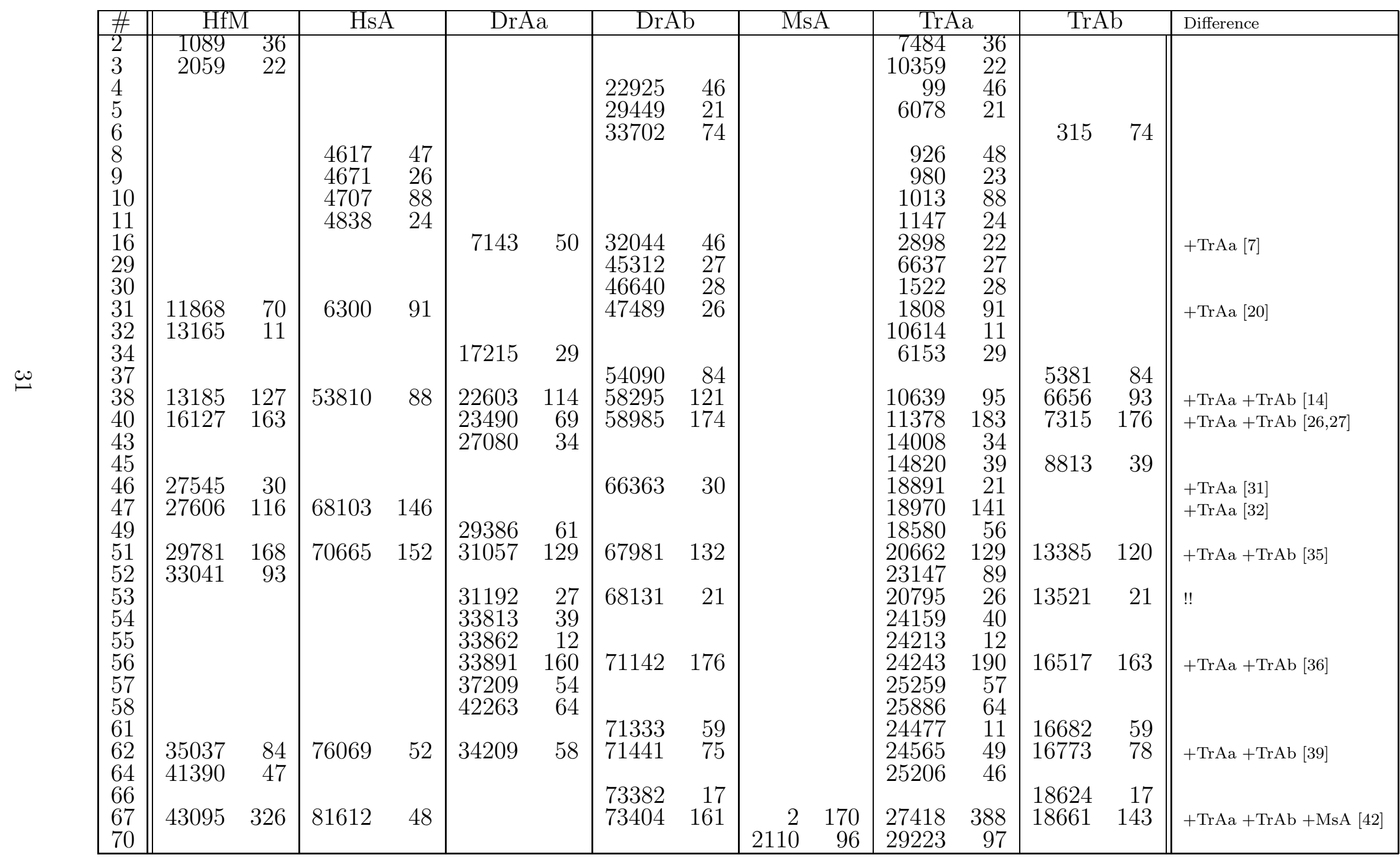


Table 13. Table 12 continued.

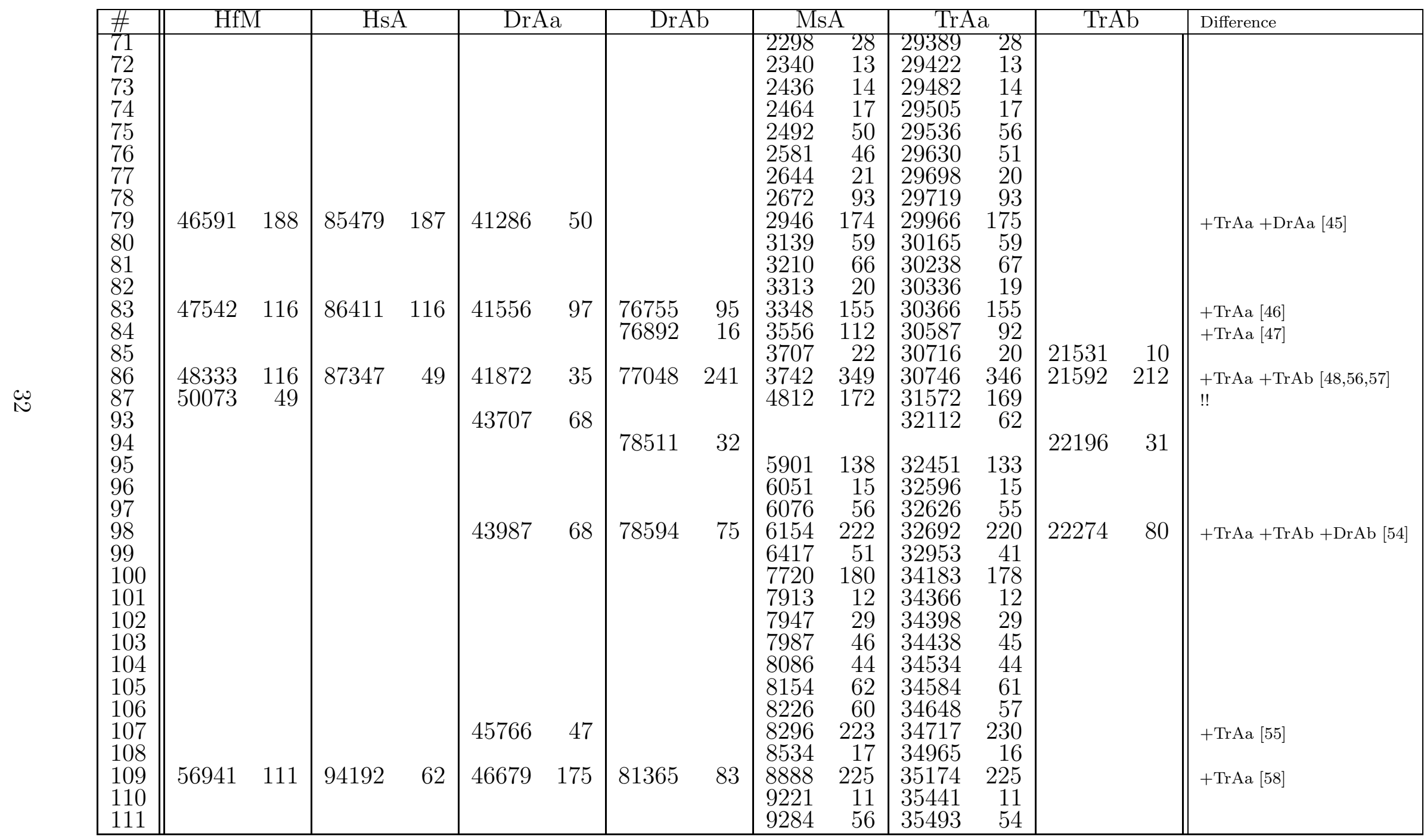


Table 14. Table 12 continued.

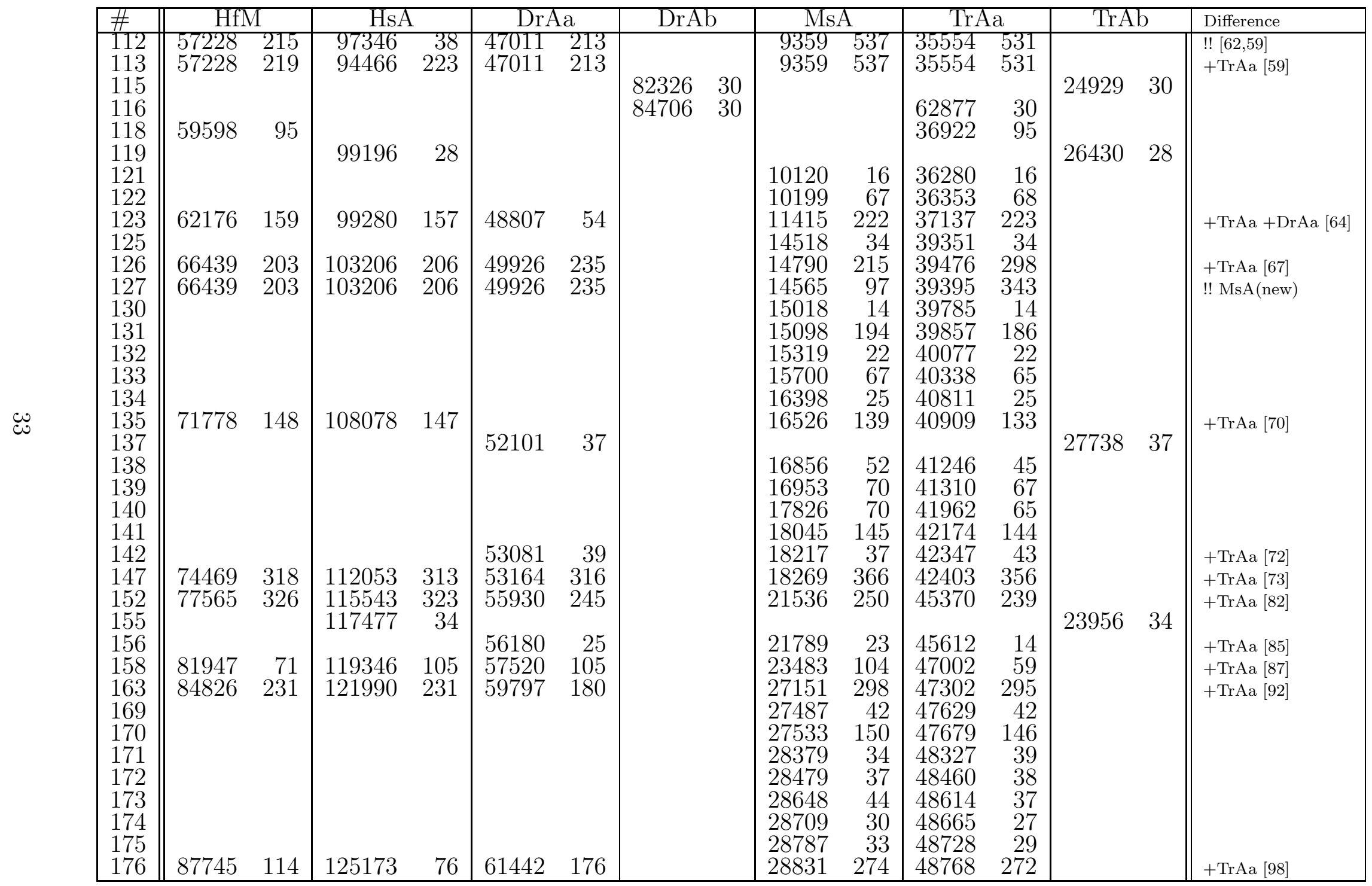


Table 15. Table 12 continued.

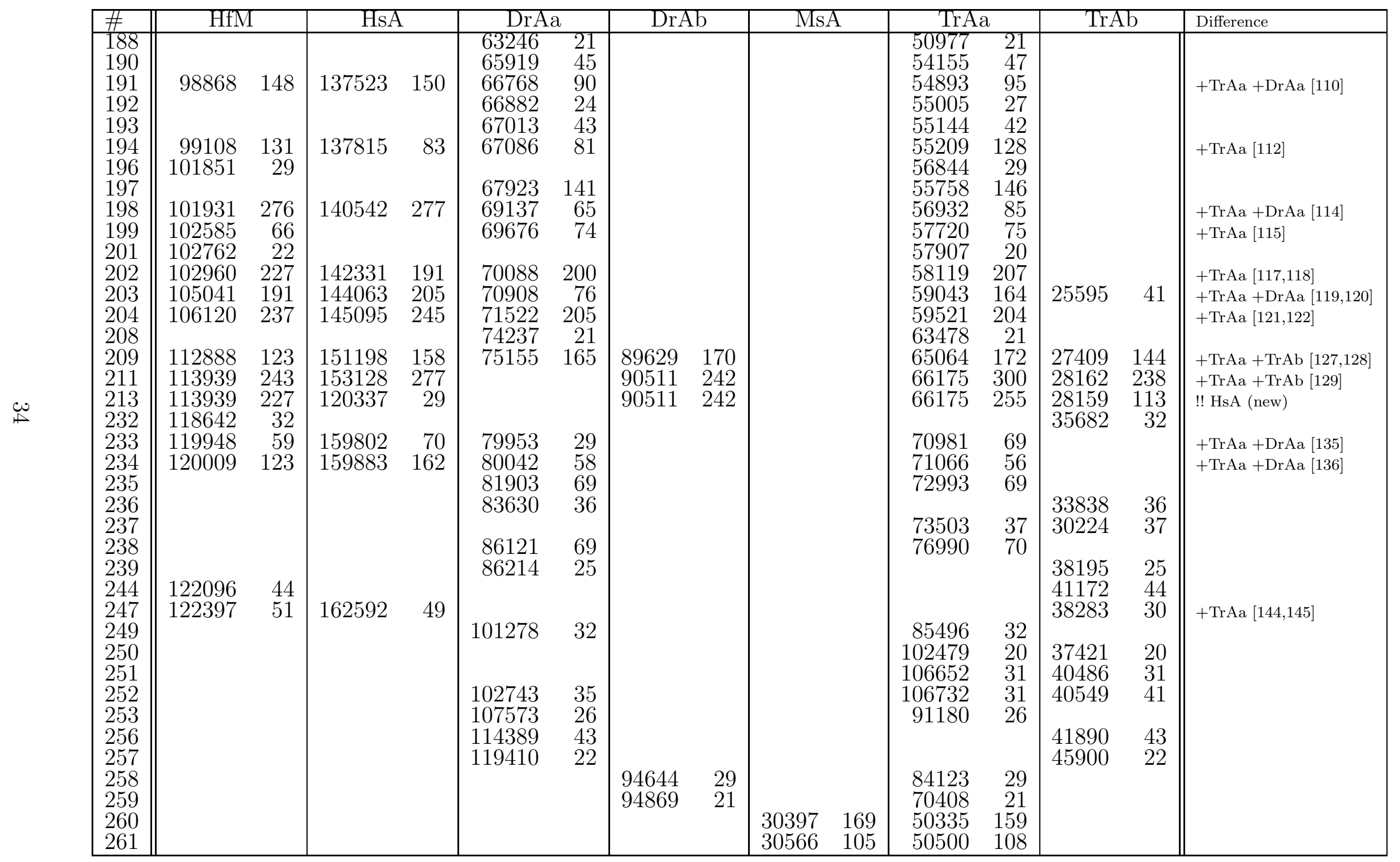

